\title{
Fingerprint Characteristic Extraction by Ridge Orientation: An Approach for a Supervised Contactless Biometric System
}

\author{
M. Kokou Assogba \\ LETIA, Ecole Polytech. \\ Ab-Calavi (EPAC) \\ 01 BP 2009 COTONOU BENIN
}

\author{
Amine Nait Ali \\ LISSI UPEC \\ 61, av Gal de GAULLE \\ 94010 Créteil France
}

\begin{abstract}
Fingerprints are the most widely used human characteristics for the purpose of people identification. However, touchbased fingerprint systems have some drawbacks due to skin elasticity, inconsistent finger placement, contact pressure, small sensing area, environment conditions and sensor noise. In this paper, we present a contactless fingerprint system based on a supervised contactless image acquisition and a ridge minutiae extraction method based on orientation computation. The fingerprint image acquisition method requires only an ordinary camera. The ridge orientation characteristic extraction is based on a double windowing of the ridge ending points and a single windowing of the ridge bifurcation points.
\end{abstract}

\section{General Terms}

Image Processing, Pattern Recognition.

\section{Keywords}

Contactless Biometry, Fingerprint characterization.

\section{INTRODUCTION}

Fingerprints are the most widely used human characteristics for people identification. A fingerprint pattern is composed by ridges and valleys. Ridges present various kinds of discontinuities (minutiae), able to capture the invariant and discriminatory information, used to recognize fingerprints. Ridge bifurcations and ridge endings (figure 1) are commonly used minutiae in Automatic Fingerprint Identification Systems (AFIS).

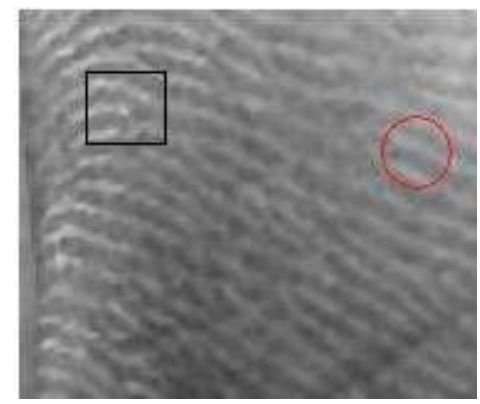

Fig 1: fingerprint features

with ridge ending in ellipse and bifurcation in square

Fingerprint sensors available today are in general touch-based for which acquisition of fingerprint images is performed by pressing or rolling fingers on a glass or plastic surface. By pressing (flat fingerprints) or rolling (rolled fingerprints) the finger, the acquired images are different from one acquisition to the other due to skin elasticity. Flat fingerprints are designed to acquire the fingertip and rolled fingerprints are designed to get more information on the fingertip. Matching algorithms are used to establish correspondences, translation and rotation between the query and the reference image. In general, the durability of a touch-based fingerprint scanner is weakened if heavily used. Additionally, problems like contagious diseases spreading make the use of touch-based scanners not very safe.

Contactless fingerprint systems are being developed as a credible alternative to overcome above problems and others related to touch-based fingerprint. The US Department of Homeland Security (DHS) considers that the development of a Biometric Detector prototype capable of acquiring contactless fingerprint for identity management will improve fingerprint acquisition quality and recognition and reduce false positives [13]. Their contactless fingerprint program is designated as a High Impact Technology Solution (HITS).

A drawback of contactless fingerprint systems is that images cannot be easily processed due to colors and they tend to acquire images with a poor contrast in the fingertip area and with a complex image background. The quality of images depends on the distance of camera to the finger.

In this paper, we present a method to overcome above drawbacks by contactless image acquisition and processing. The supervised acquisition method consists in defining a fixed template position and distance and a uniform image background such that processing is simplified. The processing method is based on image characterization using minutiae ridges (ending points and bifurcation points) orientation. Instead of measuring orientation angle with respect to horizontal, we use two concentric windows W1 and W2 to define ending point orientation and a window of size $\mathrm{W}$ to define 3 angles of bifurcation points orientation.

The paper is structured as follows. In section 2, we present our supervised contactless fingerprint system including a state of the art of existing contactless fingerprint systems. Section 3 deals with the minutiae based ridge orientation characterization. Results and discussion are presented in section 4. Section 5 concludes and presents our future works.

\section{THE SUPERVISED CONTACTLESS FINGERPRINT SYSTEM}

Historically, the acquisition of fingerprint images was performed by using the so-called "ink-technique": the subject's fingers were smeared with black ink and pressed or rolled on a paper card; the card was then scanned by using a 
general purpose scanner, producing a digital image. This kind of acquisition process is referred to as off-line sensing. Nowadays, most civil and criminal AFIS accept live-scan digital images acquired by directly sensing the finger surface with an electronic fingerprint scanner [1]. We present first the live-scan fingerprint systems and then after the existing contactless fingerprint systems, followed by our supervised contactless system.

\subsection{Touch-based Fingerprint Systems}

The most important part of a live-scan fingerprint scanner is the sensor. Existing sensors are optical, thermal, capacitive, ultrasound, electric field, piezoelectric etc. To acquire fingerprint images with those sensors, the user must press or roll his finger on a glass or plastic surface of the sensor. The pressure of the finger on the sensor platen causes some nonlinear distortions and inconsistencies in the acquired images. By the same way, the sizes of fingerprints are different from one acquisition to the other. Another drawback of the touch-based fingerprint systems is the hygienic one. The sensor platen need to be cleaned periodically not only for hygiene but also for dust as a dusty platen introduces artifacts in the acquired images.

One of the important problems of touch-based systems is that they can be deceived by submitting artificial reproductions of fingerprints made up of silicon or gelatin to the sensor. In these last years this question is the focal point of numerous research groups, both academic and industrial [2]. Some works showed the possibility of the fingerprint reproduction and defrauding of a biometric system by using Play Doh, dental impression material, plaster, silicon liquid, wax, gelatin etc [3].

More than the fraudulent use of touch-based systems, environment factors such as temperature and humidity can have negative effects on the platen of sensors.

Contactless fingerprint systems can help solving problems caused by the drawbacks mentioned above.

\subsection{Contactless Fingerprint Systems}

In order to solve the innate problems of touch-based sensors, various types of contactless sensors are being developed. Also, a large variety of algorithms have been proposed in order to achieve better authentication performance. [4] said that the touch-less fingerprint recognition is regarded as a viable alternative to contact-based fingerprint recognition technology. They used an 8 megapixel digital camera as input device for their system. The finger is not in contact with any surface but the scanner may be equipped with mechanical support to facilitate the user in presenting the finger at a uniform distance. The specified distance in the image acquisition experiment is between 4 and $6 \mathrm{~cm}$ [5].

To overcome the problem of distortions and inconsistencies on images due to pressure on live-scan platen, Touch less Biometric Systems Inc. has developed the Surround Imager $\mathrm{TM}$, a device able to capture a rolled-equivalent fingerprint without the need of touching any surface. This multi-camera system acquires different finger views that are combined to generate a $3 \mathrm{D}$ representation of the fingerprint [6]. During the capture, the finger is placed on a special support to avoid trembling that could create motion blur. The portion of the finger that has to be captured does not touch any surface. The device is a cluster of 5 cameras located on a semicircle and pointing to its center, where the finger has to be placed during the acquisition. The size of acquired images is $640 \times 480$ pixels. Since the finger has to be far away from the 5 sensors with a distance depending on the sensor size, the lens system and the required optical resolution, a distance of $50 \mathrm{~mm}$ has been fixed.

The optical system has been designed by authors to ensure a resolution of 700dpi in the center and a minimum of $500 \mathrm{dpi}$ on the image borders.

[7] presented a contactless fingerprint system working in ambient light at high resolution. The goal of the proposed method is to process in real time, each frame from the capture system.

The fingertip starts moving from $200 \mathrm{~mm}$ and stops at $100 \mathrm{~mm}$ before the lens. According to authors, a large portion of the finger image can be considered as background, since the real biometric information is only related to the ridge structures lying on the surface of the fingertip and not to the colors and the fingerprint itself. In addition, the presence of strong reflections on the skin due to the illumination/environmental light is capable to hide the real fingerprint pattern.

To overcome some drawbacks of touch-based systems and those of above contactless systems, we present a supervised contactless system.

\subsection{The Supervised Contactless}

In order to solve the innate problems of touch-based sensors, various types of contactless sensors are being developed. Some systems use simply a mobile camera. For those, the contrast between the ridges and the valleys in the images is lower than that in images obtained with touch-based sensors. Because the depth of field of the camera is small, some parts of the fingerprint regions are in focus but some parts are out of focus [8].

Whatever the contactless system is, it may happen that the ridge pattern is not sufficiently detailed, due to the fact that the finger is too far from the capture system. The obtained image can also be blurred if the finger is too close to the capture system. Also, if the finger is placed in skewed position with respect to the field view of the capture system, the important characteristics of the fingerprint are not visible.

\section{MATERIAL AND METHOD}

\subsection{Experiment Conditions}

The supervised contactless system we are presenting consists of a mean resolution camera (Nikon D80) and an illumination material as shown in figure 2 . 


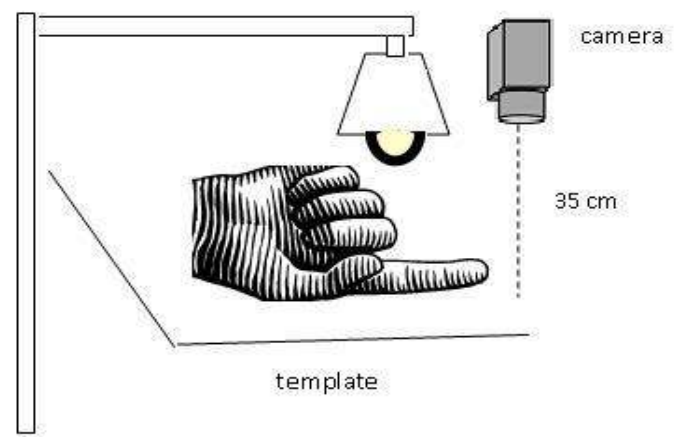

Fig 2: the supervised contacless acquisition system

The user is asked to put his finger on a template of fixed position. This position depicts the supervision process. The template is on a table and the user puts the reverse of his finger on the template and the palm faces the camera. Distance from camera to the template and resolution of image are two important parameters in contactless image acquisition. In fact, many distances have been tested and the optimum one is at $35 \mathrm{~cm}$ between the camera and the template. In our experiment, the camera ensures a resolution of 200 dpi. For comparison, table 1 presents distance values of experiments related in [5], [6], [7] and [8]. Let's denote DCS, the digital camera system of [5], SUI, the Surround Imager ${ }^{\text {TM }}$ of [6], CFS, the contactless fingerprint system of [7], MPS, the mobile camera system of [8] and SCS our supervised contactless system.

Table 1. Experiment distances comparison

\begin{tabular}{|c|c|c|c|c|c|}
\hline \hline \multicolumn{6}{|c|}{ Contactless Experiment Distances } \\
\hline \hline Experiment & DCS & SUI & CFS & MPS & SCS \\
\hline D & 50 & 50 & 200 & 60 & 350 \\
\hline Diff(d) & 300 & 300 & 150 & 290 & 0 \\
\hline
\end{tabular}

In table 1 , distance values are in $\mathrm{mm}$. Let $\mathrm{X}$ be any distance value. The difference is computed as

$$
\operatorname{Diff}(d)=|X-S C S|
$$

Various resolutions are registered in table 2 for comparison. The reference value for comparison is $500 \mathrm{dpi}$. This is the minimum resolution of scanners for FBI-compliant and is met in many commercial devices [1].

Table 2. Experiment resolutions comparison

\begin{tabular}{|c|c|c|c|c|c|}
\hline \multicolumn{6}{|c|}{ Contactless Experiment Resolutions } \\
\hline \hline Experiment & DCS & SUI & CFS & MPS & SCS \\
\hline R & 500 & 600 & 700 & 700 & 200 \\
\hline Diff(r) & 0 & 100 & 200 & 200 & 300 \\
\hline
\end{tabular}

In table 2, resolution values are in dpi. Let $\mathrm{Y}$ be any resolution value. The difference is computed as

$$
\operatorname{Diff}(r)=|Y-F B I c|
$$

where FBIc is 500 dpi, the FBI-compliant.

Even though we have 300 dpi differences for SCS compared to the FBI-compliant, we notice that the images from our experiments are clear enough and sufficiently detailed as shown in figure $4 c$ which is a zoomed ROI of figure $3 b$. The acquired images are JPEG format and have a size of $3872 \times 2592$. They are reduced for presentation in the paper.

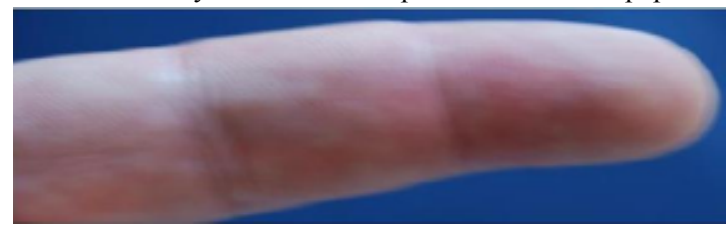

a)

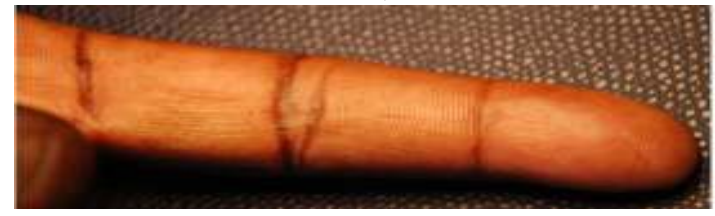

b)

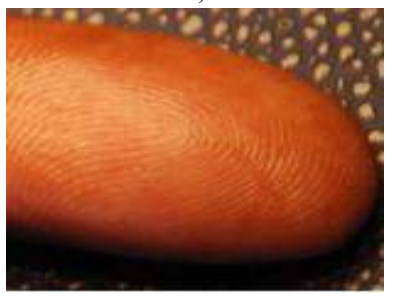

c)

Fig 3: images from SCS

As seen from figure 3 , a paramount advantage of contactless image acquisition is that a large image area can be captured quickly compared touch based systems. By acquiring the entire image of the index, we can get more information compared to flat and rolled fingerprints. Recall that index and thumbs are the most used fingerprints in authentication systems.

\subsection{Ridge Orientation Fingerprint Processing}

A fingerprint consists of two special direction-oriented parts: ridges and valleys, where valleys are the space between ridges and vice versa. These directional patterns contain various fingerprint features including a small number of singular points such as delta and core points and randomly distributed local discontinuities called minutiae [9]. Authors propose a ridge orientation estimation and verification algorithm that can not only generate an orientation of ridge flows but also verify its reliability, using directly grey level values.

In this paper, we are interested to minutiae ridges points (ridge ending points and ridge bifurcation points). As it is obvious that the contrast between the ridges and valleys of images from a contactless system is lower than that of images 
from touch based sensors, we think that the algorithm presented in [9] cannot work with the SCS images.

\subsubsection{Pre-processing}

Images from SCS experiments are Red-Green-Blue color images and they cover the entire finger. The first thing we do is to define a ROI on which we want to work. This ROI is zoomed to $128 \times 128$ or $512 \times 512$ to get the appropriate size for computation. The zoomed image is converted to grey scale image using ImageJ. In order to separate the fingerprint image from the background, a photometric adaptive threshold method has been developed [10]. Two thresholds are defined i.e. $S_{s}$ and $S_{h}$ corresponding to the mean of a square framework and the mean of a hexagonal framework.

A pixel $P(x, y)$ is deleted or not by comparing its value with $S_{s}$ and $S_{h}$. The photometric threshold is followed by a morphologic threshold to get the ridges in the image. The ridges image is skeletonized in order to get minutiae i.e. the ridge ending points and ridge bifurcation points.

\subsubsection{Ridge orientation characterization}

In general, a minutia may be described by a number of attributes, including its location in the fingerprint image, orientation, type (i.e ridge ending or ridge bifurcation), a weight based on the quality of fingerprint image in the minutia neighborhood, etc. [1]. The most used characterization considers each minutia as a triplet $\{x, y, \theta\}$ that indicates the $(\mathrm{x}, \mathrm{y})$ minutia location coordinates and the minutia orientation $\theta$. [11] and [12] used coordinates and orientations of minutiae for their characterization. In their methods, orientations are determined using horizontal axis. By this way it is clear enough that the determined orientations cannot be invariant by rotation.

In our approach, for bifurcation points we define a window $\mathrm{W}$ of size $\mathrm{SxS}$ and of central pixel the minutiae points. We count 3 points $\mathrm{P} 1, \mathrm{P} 2$, and $\mathrm{P} 3$ around the perimeter of the window as shown in figure 4 .

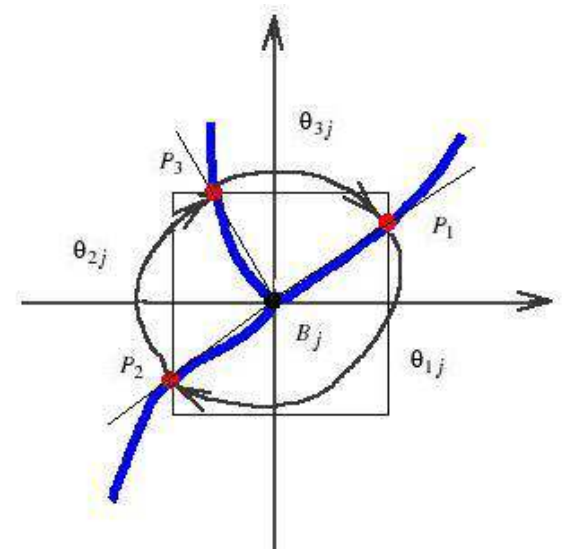

Fig 4: orientations of bifurcation points

$\mathrm{S}$ has been defined empirically to 6 pixels as the minimum length of bifurcation branches is 8 pixels.
For a given bifurcation point $\mathrm{B}_{\mathrm{j}}$, we compute the orientations as being:

$$
\begin{aligned}
& \theta_{1 j}=\operatorname{Arccos}\left(\frac{\overrightarrow{B_{j} P_{1}} \cdot \overrightarrow{B_{j} P_{2}}}{\overrightarrow{B_{j} P_{1}} \times \overrightarrow{B_{j} P_{2}}}\right) \\
& \theta_{2 j}=\operatorname{Arccos}\left(\frac{\overrightarrow{B_{j} P_{2}} \cdot \overrightarrow{B_{j} P_{3}}}{\overrightarrow{B_{j} P_{2}} \times \overrightarrow{B_{j} P 3}}\right) \\
& \theta_{3 j}=\operatorname{Arccos}\left(\frac{\overrightarrow{B_{j} P_{3}} \cdot \overrightarrow{B_{j} P_{1}}}{\overrightarrow{B_{j} P_{3}} \times \overrightarrow{B_{j} P_{1}}}\right)
\end{aligned}
$$

For ridge ending points, we define two concentric windows (W1 and W2) of central point the ridge ending point and for size S1 and S2. On the perimeter of $\mathrm{W} 1$ we have a point $\mathrm{P} 1$ and on the perimeter of $\mathrm{W} 2$, we have a point $\mathrm{P} 2$ as shown on figure 5. For a given ending point $\mathrm{Ti}$, the orientation is defined as the angle between vectors $\overrightarrow{T_{i} P_{1}}$ and $\overrightarrow{T_{i} P_{2}}$.

$\mathrm{S} 1$ has been defined empirically to 3 pixels and S2 to 6 pixels as the minimum length of the branch of ridge ending point is 8 pixels.

We have:

$\theta_{i}=\operatorname{Arccos}\left(\frac{\overrightarrow{T_{i} P_{1}} \cdot \overrightarrow{T_{i} P_{2}}}{\overrightarrow{T_{i} P_{1}} x \overrightarrow{T_{i} P_{2}}}\right)$.

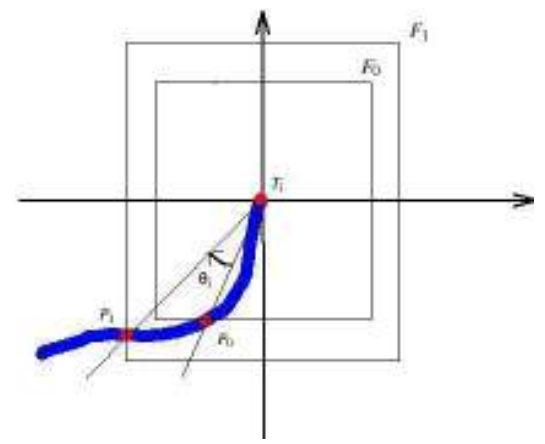

Figure 5: orientation of ridge ending points

\section{RESULTS AND DISCUSSION}

In this section, results are highlighted by illustrating our characterization method on 5 selected bifurcation points as shown in figure 6 a) and 5 selected ending points as shown in figure $6 \mathrm{~b}$ )

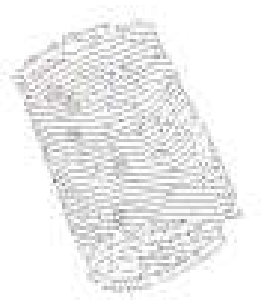

a) Ridge bifurcation points points

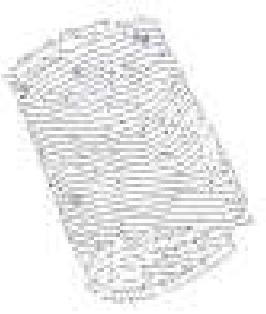

b) ridge ending
Figure 6: selected points for characterization 


\subsection{Characterization of bifurcation points}

For an image having $\mathrm{M}$ validated bifurcation points, each point $\mathrm{j}$ is represented by a quintuplet

$$
\left\{x, y, \theta_{j 1}, \theta_{j 2}, \theta_{j 3}\right\}
$$

the $\mathrm{x}$ and $\mathrm{y}$ are point $\mathrm{j}$ coordinates and the three angles, $\theta_{j 1}$, $\theta_{j 2}$, and $\theta_{j 3}$ are obtained by the concentric windows method. Table 3 presents orientation values of 5 selected bifurcation points of figure 6 a).

Table 3: ridge bifurcation points orientation values

\begin{tabular}{|c|c|c|c|c|}
\hline \hline $\mathrm{X}$ & $\mathrm{y}$ & $\theta_{1}$ & $\theta_{2}$ & $\theta_{3}$ \\
\hline \hline 131 & 129 & 174.96 & 149.35 & 35.69 \\
\hline 164 & 149 & 167.17 & 148.67 & 44.16 \\
\hline 164 & 193 & 167.17 & 159.31 & 32.91 \\
\hline 241 & 239 & 164.43 & 36.76 & 158.81 \\
\hline 243 & 313 & 168.14 & 34.38 & 157.48 \\
\hline
\end{tabular}

The orientations can be presented as a hyperbolic cosine function $f: \theta \rightarrow \cosh (\theta)=\frac{e^{\theta}+e^{-\theta}}{2}$.

We used cosine of $\theta$ instead of $\theta$ itself because the cosine is less sensitive to noise [13]. Figure 7 shows angle representation of bifurcation points of figure $6 \mathrm{a}$.

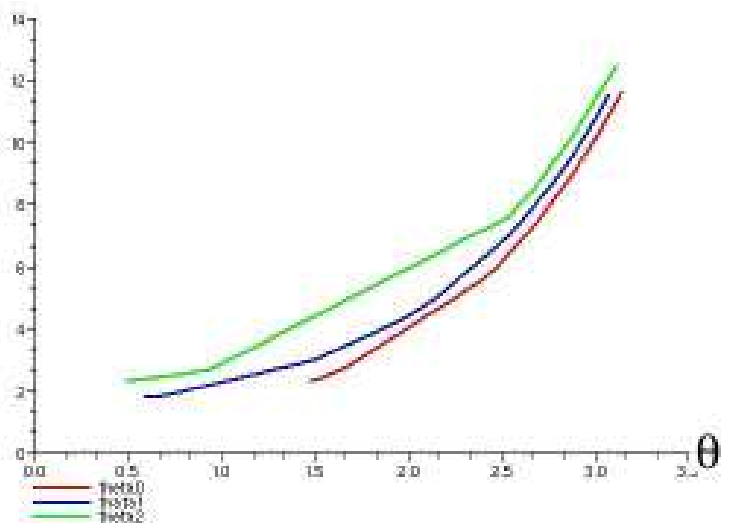

Figure 7: bifurcation points orientation representation

\subsection{Characterization of ridge ending points}

For an image having $M$ validated ending points, each ending point $\mathrm{i}$ is described by a triplet $\left\{x, y, \theta_{i}\right\}$ where $\mathrm{x}$ and $\mathrm{y}$ are point i coordinates and $\theta_{i}$ the orientation angle. Table 4 shows orientation values of selected points of figure $6 \mathrm{~b}$ ).
Table 4 orientation values of selected ending points

\begin{tabular}{|c|c|c|}
\hline \hline $\mathrm{X}$ & $\mathrm{y}$ & $\theta_{i}$ \\
\hline \hline 266 & 52 & 22.52 \\
\hline 280 & 57 & 6.48 \\
\hline 173 & 101 & 10.19 \\
\hline 93 & 126 & 13.74 \\
\hline 173 & 284 & 1.33 \\
\hline
\end{tabular}

Figure 8 shows angle representation of selected ending points of figure $6 \mathrm{~b}$.

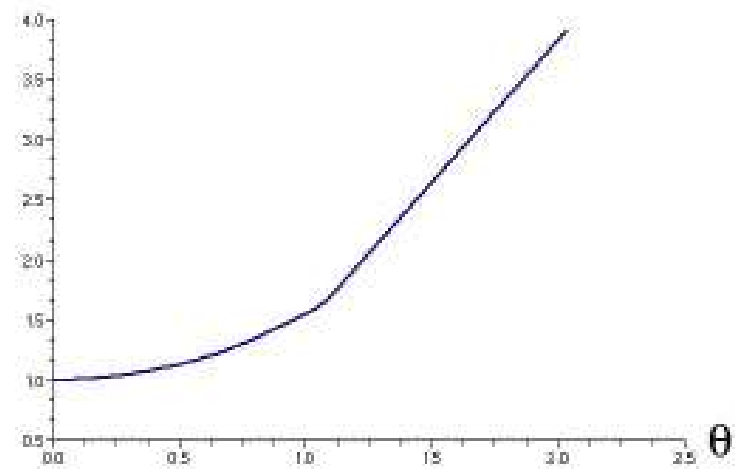

Figure 8: Selected ending points orientation function

Note that the primary aim of our project is double folded. First, we wanted to demonstrate that a contactless fingerprint system can be used to acquire the entire index image in order to have more information on individuals compared to rolled fingerprints. This goal is achieved. However, the acquired images are too big $(3872 \times 2592)$ and by this way cannot be processed in one shot. Secondly, we wanted to find an accurate representation of minutiae in contactless images such that they can be easily matched during the automatic recognition process as for existing touch-based fingerprint algorithms. We have then developed a window based ridge orientation detection method in which angles are computed with respect to crossing points of windows and not with respect to horizontal axis.

\section{CONCLUSION AND FUTURE WORK}

Fingerprints are the most widely used human characteristics for people identification. However, images from touch-based fingerprint systems are different from one acquisition to the other. Variations are caused by several factors such as nonlinear geometric distortions due to skin elasticity, inconsistent finger placement and contact pressure, small sensing area, environment conditions and sensor noise. We have then presented a contactless fingerprint system by which, not only the acquired area is very large, but also, no distortion can be observed. Moreover, we have also presented a method to characterize the fingerprint using ridges orientation, extracted without contact by a camera. In a future work, the extraction 
performance can be improved by taking into account the invariance with respect to scale.

\section{ACKNOWLEDGMENTS}

Our thanks to $\mathrm{Mr}$ Tahirou DJARA of Laboratoire d'Electrotechnique, de Télécommunications et d'Informatique Appliquée (LETIA), Ecole Polytechnique d'Abomey-Calavi (EPAC) Cotonou Benin for his contribution.

\section{REFERENCES}

[1] David, M., Dario, M., Anil, K. J., Salil, P., 2009 Handbook of Fingerprint Recognition. Second Edition, Springer-Verlag London Limited.

[2] Gian, L. M., et al. 2009 First International Fingerprint Liveness Detection Competition-LivDet 2009. ICIAP LNCS 5716, pp. 12-23, Springer - Verlag Berlin Heidelberg.

[3] Matsumoto, T., Matsumoto H., Yamada K., Hoshino S. 2002 Impact of artificial gummy fingers on fingerprint systems. Proceedings of SPIE, vol 4677, Yokohama Feb 2002.

[4] Hiew, B.Y., Teoh, A.B.J., Pang Y. H. 2007 Touch-less Fingerprint Recognition System. Proc. Workshop on Automatic Identification Advanced Technologies. Pp. 24-29, Alghero, Italy 7-8 june 2007

[5] Hiew, B.Y., Teoh, A.B.J., Yin O. S. 2010 A secure digital camera based fingerprint verification system. Journal of Visual Communication and Image Representation. Vol. 21, issue 3, pp. 219-231.

[6] Parziale, G., Santana, E-D., Hauke, R. 2006 The Surround Imager: A Multi-camera Touchless Device to acquire 3D Rolled-Equivalent Fingerprints. ICB2006, LNCS 3832, pp. 244-250

[7] Ruggero, D. L, Vincenzo, P, Neural-based Measurement of Fingerprint Images in Contactless Biometric Systems clem.dii.unisi.it

[8] Lee C., Lee S., Kim J., and Kim S.J. 2006 Preprocessing of a Fingerprint Image Captured with a Mobile Camera. ICB 2006, LNCS 3832, pp. 348-355.

[9] Liu L., Dai, T-S. 2006 Ridge Orientation Estimation and Verification Algorithm for Fingerprint Enhancement. Journal of Universal Computer Science. Vol. 12, $\mathrm{n}^{\circ} 10$, pp. 1426-1438.

[10] Djara, T., Assogba M. K., Nait Ali, A. 2010 Caractérisation spatiale des empreintes de l'index en analyse biométrique. Actes du CARI 2010. Yamoussoukro.

[11] Galy, N. 2005 Etude d'un système complet de reconnaissance d'empreintes digitales pour un capteur microsystème à balayage. Institut National Polytechnique de Grenoble - INPG.

[12] Tisse, C-L., Martin, L., Torres, L., Robert, M. 1996 Système automatique de reconnaissance d'empreintes digitales. Sécurisation de l'authentification sur carte à puce. Traitement du signal. Volume $13 \mathrm{n}^{\circ} 1$.

[13] Coty, T. 2007 Biometric Detector an S and T Innovation. Stakeholders Conference, May 21-24 2007. www.homelandsecurity.org/stakeholders 